\title{
Salep as a biological source for the synthesis of biochar with utility for the catalysis
}

\author{
Samahe Sadjadi ${ }^{1}$, Majid M. Heravi ${ }^{2}$, Fatemeh Ghoreyshi Kahangi ${ }^{3}$ \\ 1- Gas Conversion Department, Faculty of Petrochemicals, Iran Polymer and Petrochemicals Institute, \\ Tehran, Iran \\ 2- Department of Chemistry, School of Science, Alzahra University, Tehran, Iran \\ 3- Department of Chemistry, University Campus 2, University of Gilan, Rasht, Iran
}

\begin{abstract}
Hydrothermal carbonization of salep as a domestic biosource was carried out to afford a biochar, BC, with exceptional catalytic activity. BC can be further magnetized by incorporation of magnetic nanoparticles to furnish a magnetic catalyst, $\mathrm{BC}-\mathrm{Fe}$, with improved recovery and recyclability for the hydrogenation of nitroarenes in the absence of any precious metal. BC-Fe was also applied as a catalyst support for the immobilization of Pd nanoparticles and development of an efficient, biocompatible and cost-effective catalyst, Pd@BC-Fe, with utility for the oxidation of benzyl alcohols under mild reaction condition in a selective manner to afford corresponding acids in high yields. The study of the recyclability of the catalyst confirmed high recyclability of Pd@BC-Fe.
\end{abstract}

Keywords: biochar, heterogeneous, catalysts, hydrogenation, oxidation, salep 